\title{
A non-inferiority study to analyze the safety of totally tubeless percutaneous nephrolithotomy
}

\author{
Piotr Bryniarski ${ }^{A-F}$, Paweł Rajwa ${ }^{\mathrm{B}}$, Marcin Życzkowski ${ }^{\mathrm{C}}$, Piotr Taborowski ${ }^{\mathrm{D}}$, Zbigniew Kaletka ${ }^{\mathrm{E}}$, Andrzej Paradysz ${ }^{\mathrm{E}, \mathrm{F}}$ \\ Department of Urology, Medical University of Silesia, Katowice, Poland \\ A - research concept and design; B - collection and/or assembly of data; $C$ - data analysis and interpretation; \\ $D$ - writing the article; $E$ - critical revision of the article; $F$ - final approval of the article
}

Address for correspondence

Piotr Bryniarski

E-mail: piotr.bryniarski@hotmail.com

Funding sources

None declared

Conflict of interest

None declared

Received on January 18, 2017

Reviewed on March 28, 2017

Accepted on May 9, 2017

DOI

10.17219/acem/71196

\section{Copyright}

Copyright by Author(s)

This is an article distributed under the terms of the

Creative Commons Attribution Non-Commercial License

(http://creativecommons.org/licenses/by-nc-nd/4.0/)

\section{Abstract}

Background. Totally tubeless percutaneous nephrolithotomy (ttPCNL) becomes increasingly frequently utilized in the treatment of kidney stones. This procedure emerged as an answer for patients' needs to minimize hospitalization time, pain intensity and discomfort due to nephrostomy tube. However, ttPCNL may be less safe for patients, as without nephrostomy tube bleeding from renal vessels is potentially more severe.

Objectives. The purpose of our study was to retrospectively evaluate the safety parameters of ttPCNL collected in a prospective manner.

Material and methods. This was a single tertiary care center, non-inferiority study with 2 arms (55 patients in each arm). The $1^{\text {st }}$ group consisted of patients who underwent ttPCNL with the application of TachoSil ${ }^{\circledR}$ (Takeda, Osaka, Japan) as sealing material, while in the $2^{\text {nd }}$ group, conventional PCNL with nephrostomy tube (CPCNL) was utilized. The primary goal was to prove that hemoglobin drop after surgery, as equivalent of safety, was not inferior than $1 \mathrm{~g} / \mathrm{dL}$. The secondary endpoints comprised visual analogue scale (VAS) of pain, additional pain treatment and hospital stay.

Results. The mean hemoglobin drop after ttPCNL was insignificantly lower in comparison with CPCNL group (mean: $-0.35 \mathrm{~g} / \mathrm{dL}$; confidence interval $(\mathrm{Cl})$ : $-0.8,0.21$ ). Visual analogue scale of pain and pain treatment were comparable between groups. Hospital stay was significantly shorter in the ttPCNL group.

Conclusions. Totally tubeless PCNL can be considered a safe option after uncomplicated lithotripsy - what is important, it is characterized by a shorter hospitalization time. Postoperatively, pain intensity is comparable between both groups.

Key words: nephrolithiasis, lithotripsy, kidney stones, totally tubeless percutaneous nephrolithotomy 


\section{Introduction}

Percutaneous nephrolithotomy (PCNL) is considered a standard procedure in the treatment of kidney stones larger than $2 \mathrm{~cm}$ in diameter. ${ }^{1}$ It is also recommended in the case of smaller stones in patients with contraindications for shockwave lithotripsy (SWL), such as shockwave resistant stones and anatomical malformations, or when a patient elects PCNL as a procedure of higher efficacy.

Classic PCNL consists of 4 major steps, including the insertion of ureteral catheter, percutaneous access to appropriate kidney, lithotripsy, and protection of controlled kidney injury with nephrostomy tube. However, many variants of classic approach have been introduced recently, including supine position PCNL, tubeless PCNL (tPCNL) and totally tubeless PCNL (ttPCNL). The $1^{\text {st }}$ variant makes the procedure faster (as there is no need to rotate the patient on a table) and easier to evacuate all debris after lithotripsy. Tubeless PCNL and ttPCNL were proposed to minimize the inconvenience due to nephrostomy tube and ureteral catheter. However, there are certain contraindications for ttPCNL, including the presence of residual stones and the need for a second look, significant intraoperative bleeding, urine extravasation, ureteral obstruction, persistent bacteriuria due to infected stones, solitary kidney, bleeding diathesis, or planned percutaneous chemolitholysis. ${ }^{1}$

In order to assess the safety of ttPCNL in comparison with conventional PCNL with nephrostomy tube (cPCNL), we conducted a non-inferiority study. The aim of such a trial was to show that new treatment is not (much) worse than the standard treatment.

\section{Material and methods}

\section{Compliance with ethical standards}

The study has been performed in accordance with the ethical standards laid down in the 1964 Declaration of Helsinki. Informed consent was obtained from all individual participants included in the study.

\section{Inclusion and exclusion criteria}

Retrospective analysis of patients' records, which have been collected prospectively, was conducted; it included the period between February 2014 and December 2016. A total of 110 patients were analyzed (Fig. 1). The inclusion criteria consisted of:

- patients with stone or stones over $2 \mathrm{~cm}$ in diameter in a kidney;

- patients with stones $1.5-2 \mathrm{~cm}$ in diameter who wished to have PCNL procedure instead of SWL or retrograde intrarenal surgery;

- patients with stones $1.5-2 \mathrm{~cm}$ in diameter with contraindications for SWL.

All patients had a contrast-enhanced computed tomography $(\mathrm{CT})$ performed before planned surgery. Based on the CT results, a detailed preoperative analysis of anatomical characteristics was conducted and surgery was planned, including punctured calyx, number of accesses, inter/infra costal entry, and potential injury of adjacent organs.

Exclusion criteria consisted of:

- presence of residual stones after lithotripsy and the need for a second look;
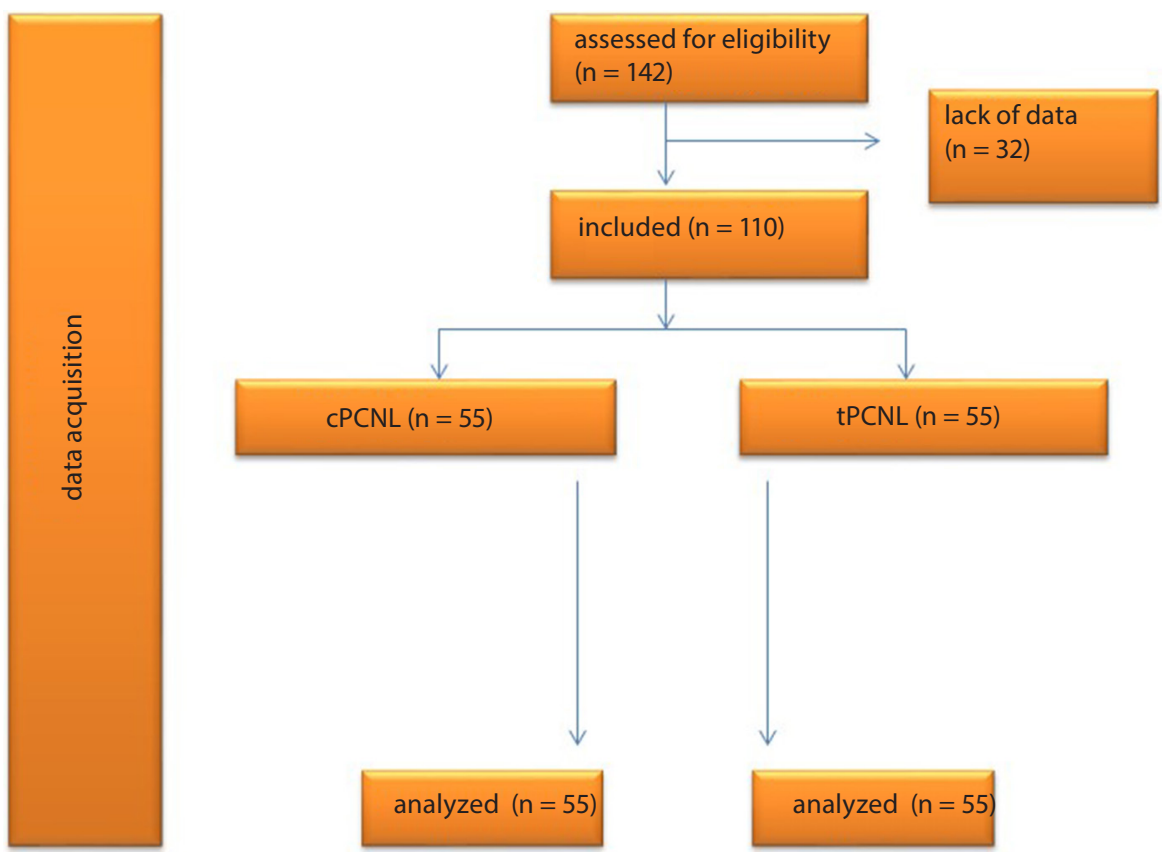

Fig. 1. Study design 
Table 1. Demographic and clinical characteristics of the analyzed groups of patients

\begin{tabular}{|c|c|c|c|c|}
\hline \multicolumn{2}{|c|}{ Parameter } & CPCNL $(n=55)$ & $\operatorname{ttPCNL}(n=55)$ & $\mathrm{p}$-value \\
\hline \multicolumn{2}{|c|}{ Age [years], median (IQR) } & $49.5(45.2-62.8)$ & $52.6(42.4-58.3)$ & 0.32 \\
\hline Sex, n (\%) & $\begin{array}{c}\text { female } \\
\text { male }\end{array}$ & $\begin{array}{l}25(45.4) \\
30(54.5)\end{array}$ & $\begin{array}{c}27(49) \\
28(50.9)\end{array}$ & 0.7 \\
\hline \multicolumn{2}{|c|}{ BMI $\left[\mathrm{kg} / \mathrm{m}^{2}\right]$, mean (SD) } & $23.1(4.3)$ & $22.3(4.5)$ & 0.45 \\
\hline
\end{tabular}

CPCNL - classic percutaneous nephrolithotomy; ttPCNL - totally tubeless percutaneous nephrolithotomy; BMI - body mass index; IQR - interquartile range; SD - standard deviation.

- massive bleeding requiring termination of the procedure;

- urine extravasation after completion of lithotripsy in fluoroscopy (renal pelvis rupture);

- persistent bacteriuria due to infected stones;

- ureteral obstruction;

- solitary kidney;

- contrast, paracetamolum and tramadol allergy;

- lack of any relevant data in our data system.

\section{Endpoints and sample size estimation}

Our primary endpoint was postoperative hemoglobin drop. The secondary endpoints comprised hospitalization time, degree of pain postoperatively and additional pain treatment. According to previous studies, an average hemoglobin drop after classic and tubeless PCNL is $0.97 \mathrm{~g} / \mathrm{dL}$ and $0.82 \mathrm{~g} / \mathrm{dL}$ (standard deviation $(\mathrm{SD})=1.54$ ), respectively. ${ }^{2,3}$ This data is taken from studies where PCNL is conducted in a different way than in the present study (e.g., TachoSil ${ }^{\circledR}$ and re-entry Malecot catheter (Coloplast, Humlebæk, Denmark) are not applied. In order to estimate the sample size for non-inferiority study, one must choose the largest difference ("delta") between means that are clinically acceptable. The upper limit of one-sided 97.5\% confidence interval (CI) for non-inferiority test ("delta") was arbitrary set for $1 \mathrm{~g} / \mathrm{dL}$. The sample size was estimated to be 47 patients per arm. The total sample size required 94 patients to achieve $95 \%$ power with 0.025 type I error. ${ }^{4-6}$

\section{Classic and tubeless procedure}

Patients were admitted to the hospital the day before the procedure. Kidney stones were diagnosed based on contrast-enhanced CT, which was performed 1 month before the planned surgery. Basic serum and urine analyses were performed, as well as an X-ray of abdomen. On the day of the surgery, patients were given prophylactic antibiotics ( 1 dose of $2^{\text {nd }}$ generation iv. cefalosporin). Patients were operated in general anesthesia in prone position. Access to pyelocalyceal system was conducted under fluoroscopy by urologist with Amplatz dilators (Cook Medical, Bloomington, USA). The access sheath had a diameter of $28 \mathrm{Fr}$ (French catheter scale) and the nephroscope was $26 \mathrm{Fr}$. After lithotripsy, in cPCNL, re-entry Malecot nephrostomy was inserted into pyelocalyceal system and ureteral catheter was withdrawn. ${ }^{7}$ In ttPCNL variant, rolled TachoSil $^{\circledR}$ was inserted through access sheath and served as a sealing material. ${ }^{8}$ After $\mathrm{cPCNL}$, nephrostomy tube was withdrawn at day 5 after the operation and if there was no significant urine leakage, patients were discharged home. After ttPCNL, if there were no complications (such as pain, fever or significant hematocrit drop), patients were discharged home on day 2. Antinociceptive treatment included $1 \mathrm{~g}$ of iv. paracetamolum every $6 \mathrm{~h}$ on the day of surgery. In addition, $100 \mathrm{mg}$ of tramadol was given intramuscularly on demand.

\section{Statistical analysis}

The study was designed as a non-inferior one to see whether the hemoglobin drop after ttPCNL (as equivalence of safety) is not higher in comparison with CPCNL. ${ }^{9-11}$ To prove non-inferiority, it must be shown that the CI of the difference between means does not include predefined "delta". In other words, if there is truly no difference between the $\mathrm{PCNL}$ and ttPCNL, then 94 patients are required to be $95 \%$ sure that the upper limit of a one-sided 97.5\% CI (or equivalently a 95\% 2-sided CI) will be below the non-inferiority limit of $1 \mathrm{~g} / \mathrm{dL}$. Usually, non-inferiority trials are conducted prospectively, but a recent study indicated that data collected retrospectively is equally valid. ${ }^{12,13}$ Therefore, we decided to perform a non-inferiority study of data collected retrospectively, but in a prospective way. In addition, we decided that if non-inferiority had been proven, we would have conducted superiority analysis. As this was the case, superiority testing comprised Student's t-test for continuous variables with normal distribution. For continuous variables without normal distribution, a Mann-Whitney U test was used. For categorical variables, a $\chi^{2}$ test was used. A p-value $<0.05$ was considered significant. All analyses were conducted using STATISTICA v. 13 (StatSoft Inc., Tulsa, USA).

\section{Results}

Demographic and clinical characteristics are given in Table 1. Patients did not differ significantly in terms of preoperative characteristics (Table 2). In the analysis, the difference between means of hemoglobin drop in 2 groups was $-0.35 \mathrm{~g} / \mathrm{dL}(\mathrm{CI}:-0.8,0.21)$. As the upper 
Table 2. Preoperative characteristics of the analyzed groups of patients

\begin{tabular}{|c|c|c|c|c|}
\hline \multicolumn{2}{|l|}{ Parameter } & $\mathrm{cPCNL}(\mathrm{n}=55)$ & $\operatorname{ttPCNL}(n=55)$ & $\mathrm{p}$-value \\
\hline \multicolumn{2}{|l|}{ Stone diameter [cm], mean (SD) } & $2.3(0.8)$ & $2.4(0.7)$ & 0.36 \\
\hline Side, n (\%) & $\begin{array}{l}\text { right } \\
\text { left }\end{array}$ & $\begin{array}{l}24(43.6) \\
31(56.3)\end{array}$ & $\begin{array}{l}29(52.7) \\
26(47.2)\end{array}$ & 0.34 \\
\hline \multicolumn{2}{|l|}{ Hemoglobin [g/dL], mean (SD) } & $14.4(1.5)$ & $14.7(1.3)$ & 0.9 \\
\hline \multicolumn{2}{|l|}{ Hematocrit [\%], median (IQR) } & $42.1(31.8-52.3)$ & $42.2(32.2-53.8)$ & 0.76 \\
\hline \multicolumn{2}{|l|}{ Creatinine $[u m o l / L]$, mean $(S D)^{*}$} & $73.1(16.2)$ & $80.6(15.7)$ & 0.16 \\
\hline \multicolumn{2}{|l|}{ VAS [points], median (IQR)* } & $1.2(0.7-1.6)$ & $1.1(0.7-1.5)$ & 0.65 \\
\hline
\end{tabular}

CPCNL - classic percutaneous nephrolithotomy; ttPCNL - totally tubeless percutaneous nephrolithotomy; SD - standard deviation; IQR - interquartile range; VAS - visual analogue scale; *assessed on the day of submission to the hospital.

Table 3. Postoperative characteristics of the analyzed groups of patients

\begin{tabular}{|c|c|c|c|c|}
\hline \multicolumn{2}{|c|}{ Parameter } & cPCNL group $(n=55)$ & ttPCNL group $(n=55)$ & p-value \\
\hline \multicolumn{2}{|l|}{ OR time [min], mean (SD) } & $63.4(23.3)$ & $66.2(24.8)$ & 0.43 \\
\hline \multicolumn{2}{|l|}{ Hemoglobin [g/dL], mean (SD)* } & $12.9(2.1)$ & $13.6(2.2)$ & 0.51 \\
\hline \multicolumn{2}{|l|}{ Hematocrit [\%], median (IQR) } & $35.6(28.2-42.9)$ & $37.1(27.9-45.6)$ & 0.31 \\
\hline \multicolumn{2}{|l|}{ Hemoglobin drop, mean (SD) } & $1.5(1.2)$ & $1.1(0.9)$ & 0.62 \\
\hline \multicolumn{2}{|c|}{ Hematocrit drop [\%], median (IQR) } & $6.5(4.3-7.9)$ & $5.1(4.5-8.2)$ & 0.41 \\
\hline \multicolumn{2}{|l|}{ Creatinine [umol/L], mean (SD)* } & $77.7(17.2)$ & $84.7(18.2)$ & 0.24 \\
\hline Blood transfusion, n (\%) & $\begin{array}{l}\text { yes } \\
\text { no }\end{array}$ & $\begin{array}{c}3(6) \\
47(94)\end{array}$ & $\begin{array}{c}2(4) \\
48(96)\end{array}$ & 0.64 \\
\hline \multicolumn{2}{|l|}{ VAS [points], median $(\mathrm{IQR})^{* *}$} & $5.6(3.1-6.2)$ & $3.5(3.4-6.8)$ & 0.12 \\
\hline \multicolumn{2}{|l|}{ Tramadol [mg], mean $(\mathrm{SD})^{* * *}$} & $312.3(202.5)$ & $277.4(166.5)$ & 0.07 \\
\hline Fever $>38^{\circ} \mathrm{C}, \mathrm{n}(\%)$ & $\begin{array}{l}\text { yes } \\
\text { no }\end{array}$ & $\begin{array}{c}5(10) \\
45(90)\end{array}$ & $\begin{array}{c}2(4) \\
48(96)\end{array}$ & 0.23 \\
\hline \multicolumn{2}{|l|}{ Hospital stay [days], median (IQR) } & $5.3(3.2-7.3)$ & $2.4(1.1-3.8)$ & 0.02 \\
\hline Supracostal access, n (\%) & $\begin{array}{l}\text { yes } \\
\text { no }\end{array}$ & $\begin{array}{l}36(72) \\
14(28)\end{array}$ & $\begin{array}{l}37(74) \\
13(26)\end{array}$ & 0.82 \\
\hline Number of accesses, n (\%) & $\begin{array}{c}>1 \\
1\end{array}$ & $\begin{array}{c}9(18) \\
41(82)\end{array}$ & $\begin{array}{c}7(14) \\
43(86)\end{array}$ & 0.58 \\
\hline $\begin{array}{l}\text { Residual stones in renal pelvis, } \\
\mathrm{n}(\%)^{* * * *}\end{array}$ & $\begin{array}{l}\text { yes } \\
\text { no }\end{array}$ & $\begin{array}{c}5(10) \\
45(90)\end{array}$ & $\begin{array}{c}3(6) \\
47(94)\end{array}$ & 0.46 \\
\hline DJ insertion, $n(\%)^{* * * * *}$ & $\begin{array}{l}\text { yes } \\
\text { no }\end{array}$ & $\begin{array}{c}3(6) \\
47(94)\end{array}$ & $\begin{array}{c}6(12) \\
44(88)\end{array}$ & 0.29 \\
\hline
\end{tabular}

CPCNL - classic percutaneous nephrolithotomy; ttPCNL - totally tubeless percutaneous nephrolithotomy; OR - operating room; SD - standard deviation; IQR - interquartile range; VAS - visual analogue scale; DJ - double-J ureteral catheter; * blood sample collected $5 \mathrm{~h}$ after the procedure; ** obtained 1 day after the procedure; ${ }^{* *}$ throughout hospital stay; ${ }^{* * *}$ abdominal X-ray the day after the procedure - stone debris $<3$ mm also classified as "yes"; ***** in ttPCNL - after the procedure, in CPCNL - after nephrostomy tube removal.

limit of CI (0.21) is lower than the predefined "delta" $(1 \mathrm{~g} / \mathrm{dL})$, the non-inferiority of ttPCNL was proven.

In superiority analysis, there were no differences between groups in terms of safety (Table 3). However, hospitalization time was shorter in case of ttPCNL groups. The multivariate analysis of serum creatinine levels (Hotelling's test) did not show differences before and after the procedure between groups $(\mathrm{p}<0.37)$. Other parameters, such as postoperative visual analogue scale (VAS) of pain and additional tramadol treatment, were comparable between groups.

In both groups, small debris was diagnosed in abdominal $\mathrm{X}$-ray the day after the procedure, though fluoroscopy at the end of procedure revealed stone-free status. It is worth mentioning that all these residual stones were $<4 \mathrm{~mm}$ in diameter.

Complications occurred only in a few patients. We observed arteriovenous fistula in 1 patient in the ttPCNL group. Pleural injury occurred in 1 patient in the cPCNL group. There were no colon injuries in our cohort.

\section{Discussion}

Conventional PCNL is the standard treatment for kidney stones over $2 \mathrm{~cm}$ in diameter. It is a highly effective treatment with a success rate over $90 \%$. Because such a high efficacy was achieved, the next step for CPCNL was to limit 
the trauma associated with procedure rather than further increase success rates. One of these attempts was to eliminate nephrostomy tube as it contributes to flank pain during hospitalization. Hence, other methods were sought to compensate for nephrostomy tube. ${ }^{14}$ Among them are sealing materials, including human fibrinogen - TachoSil ${ }^{\circledR} \cdot{ }^{15}$ After lithotripsy, it may be located within the tract in kidney parenchyma. It assures very good hemostasis and prevents urine leakage after a tubeless procedure. However, its safety was not established (so far) after each procedure; therefore, it may be utilized only without contraindications (above-mentioned). The $2^{\text {nd }}$ step to decrease the pain after PCNL was to eliminate ureteral catheter. ${ }^{16,17}$ The reason to utilize them was to prevent renal colic after the procedure as small debris or blood clots may fall into ureter. On the other hand, it also contributes to flank discomfort. As we have shown, $12 \%$ of patients may require double-J ureteral catheter (DJ) insertion due to renal colic after lithotripsy. It is highly probable that after lithotripsy, small debris or blood clots from kidney move to the ureter and cause flank pain. In our opinion, it is better to insert DJ catheter when it is really necessary than leave it in place after PCNL routinely, especially because after excluding patients with renal colic $(n=9)$, VAS was favorable for ttPCNL (Table 3). Six (12\%) patients had renal colic the day after lithotripsy in comparison with only $3(6 \%)$ patients in the cPCNL group; hence, the median of VAS was not favorable for the ttPCNL group. Colic in cPCNL group occurred due to nephrostomy obstruction with clots, which required irrigations or nephrostomy replacement. We have to emphasize that after both CPCNL (where re-entry Malecot nephrostomy is utilized) and ttPCNL we always remove ureteral catheter at the end of the procedure.

Many trials were publicized with an effort to prove safety of tPCNL and ttPCNL. Thanks to those pioneer authors, both of these procedures are today commonly conducted in many urological centers. However, the quality of evidence that both these new treatments are equally safe as cPCNL is still unsatisfactory. All those studies were superiority trials with the aim to prove that experimental treatment is superior to standard treatment. ${ }^{18-20}$ In statistical language, the aim was to reject null hypothesis (experiment is not better than standard) and adopt an alternative one (experiment is better than standard). In the above-mentioned trials, statistical significance was not shown ( $\mathrm{p}>0.05$ ); hence, the authors stated that ttPCNL are equally safe as $\mathrm{CPCNL}$. Although this statement is true (which we proved in our trial), it is also based on the misconception of statistical results. It is worth mentioning that the phrase "experiment is not better than standard" is not the same as "experiment and standard are equal". ${ }^{6}$ To prove that the new treatment is not (much) worse than the standard treatment, a non-inferiority trial has to be conducted. The major flaw of these trials is the need to adopt the smallest difference between interventions that is clinically significant. In our study, it was the difference between mean hemoglobin drop after 2 procedures. It is very difficult to say what difference would be clinically significant. Hemoglobin drop which leads to blood transfusion might be considered clinically significant. However, we perform transfusions when the hemoglobin concentration is lower than $8 \mathrm{~g} / \mathrm{dL}$. The average hemoglobin concentration after PCNL was $13.4 \mathrm{~g} / \mathrm{dL}$, so the clinically significant difference should be $5.4 \mathrm{~g} / \mathrm{dL}$. Such a huge difference would be unacceptable and it surely could not be stated that both treatments are equally safe. On the other hand, it is acceptable when the difference in hemoglobin drop after the 2 procedures is within the limit of $1 \mathrm{~g} / \mathrm{dL}$.

If non-inferiority was proven, it is reasonable to ask whether ttPCNL is safer than $\mathrm{PCNL}$, especially because direct difference is favorable for ttPCNL (hemoglobin drop $1.1 \mathrm{~g} / \mathrm{dL}$ vs $1.5 \mathrm{~g} / \mathrm{dL})$. Such a small difference, even if superiority was proven, would bring nothing to the practice. However, hospitalization time was favorable for ttPCNL group.

\section{Conclusions}

1. We are at least $95 \%$ sure that in the whole population of patients after ttPCNL, the mean difference in hemoglobin drop in comparison with cPCNL is smaller than $1 \mathrm{~g} / \mathrm{dL}$. That makes this procedure equally safe as CPCNL.

2 . We could not prove that ttPCNL has a favorable outcome in terms of intensity of pain postoperatively.

3. Occasionally, ureteral catheter insertion may be needed after ttPCNL. After excluding subjects with renal colic, patients from ttPCNL group experience lower pain postoperatively.

4. Totally tubeless PCNL allows shorter hospitalization time.

\section{References}

1. Türk C, Petřík A, Sarica K, et al. EAU guidelines on interventional treatment for urolithiasis. Eur Urol. 2016;69(3):475-482.

2. Shah H, Khandkar A, Sodha H, Kharodawala S, Hegde S, Bansal M. Tubeless percutaneous nephrolithotomy: 3 years of experience with 454 patients. BJU Int. 2009;104(6):840-846.

3. Wang J, Zhao C, Zhang C, Fan X, Lin Y, Jiang Q. Tubeless vs standard percutaneous nephrolithotomy: A meta-analysis. BJU Int. 2012;109(6): 918-924.

4. Online calculator: Compare 2 Means 2-Sample Non-Inferiority or Superiority. http://powerandsamplesize.com/Calculators/Compare2-Means/2-Sample-Non-Inferiority-or-Superiority. Accessed December 10, 2016.

5. Chow S, Shao J, Wang H. Sample Size Calculations in Clinical Research. $2^{\text {nd }}$ ed. Boca Raton, FL: Chapman \& Hall/CRC Biostatistics Series; 2008.

6. Lesaffre E. Superiority, equivalence, and non-inferiority trials. Bull NYU Hosp Jt Dis. 2008;66(2):150-154.

7. Liatsikos EN, Hom D, Dinlenz CZ, et al. Tail stent versus re-entry tube: A randomized comparison after percutaneous stone extraction. Urology. 2002;59:15-19.

8. Bryniarski P, Bogacki R, Muskała B, Taborowski P, Paradysz A. Tubeless percutaneous nephrolithotomy. Videourology. 2016;30:vid.2016.0023. doi:10.1089/vid.2016.0023

9. Pocock SJ. The pros and cons of noninferiority trials. Fundam Clin Pharmacol. 2003;17(4):483-490. 
10. D'Agostino RB, Massaro JM, Sullivan LM. Non-inferiority trials, design concepts and issues - the encounters of academic consultants in statistics. Stat Med. 2003;22(2):169-186.

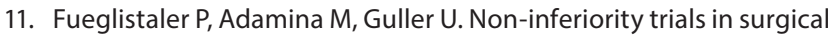
oncology. Ann Surg Oncol. 2007;14(5):1532-1539.

12. Patel $A B$, Reyes $A$, Ackland GL. Non-inferiority of retrospective data collection for assessing perioperative morbidity. PeerJ. 2015;3:e1466

13. Deng C, Chen J, Hanna K. Retrospective assessment of non-inferiority in the rare disease, Guillain-Barre syndrome. Biom Biostat Int J. 2014;1(2):00009. doi:10.15406/bbij.2014.01.00009

14. Lee DI, Uribe C, Eichel L, et al. Sealing percutaneous nephrolithotomy tracts with gelatin matrix hemostatic sealant: Initial clinical use. J Urol. 2004;171:575-578.

15. Cormio L, Perrone A, Di Fino G, et al. TachoSil $\left({ }^{(\mathbb{R}}\right)$ sealed tubeless percutaneous nephrolithotomy to reduce urine leakage and bleeding: Outcome of a randomized controlled study. JUrol. 2012;188(1):145-150.
16. Karami H, Gholamrezaie HR. Totally tubeless percutaneous nephrolithotomy in selected patients. J Endourol. 2004;18(5):475-476.

17. Aghamir SM, Hosseini SR, Gooran S. Totally tubeless percutaneous nephrolithotomy. J Endourol. 2004;18(7):647-648.

18. Yates DR, Safdar RK, Spencer PA, Parys BT. "Nephrostomy-free" percutaneous nephrolithotomy: Experience in a UK district general hospital. Ann R Coll Surg Engl. 2009;91(7):570-577.

19. Mishra S, Sabnis RB, Kurien A, Ganpule A, Muthu V, Desai M. Questioning the wisdom of tubeless percutaneous nephrolithotomy (PCNL): A prospective randomized controlled study of early tube removal vs tubeless PCNL. BJU Int. 2010;106:1045-1048.

20. Li R, Louie MK, Lee HJ, et al. Prospective randomized trial of three different methods of nephrostomy tract closure after percutaneous nephrolithotripsy. BJU Int. 2011;107:1660-1665. 